9. McBride A, Chau TTH, Hong NTT, Mai NTH, Anh NT, Thanh TT, et al. Angiostrongylus cantonensis is an important cause of eosinophilic meningitis in southern Vietnam. Clin Infect Dis. 2017;64:1784-7. http://dx.doi.org/10.1093/cid/cix118

Address for correspondence: Damien K.Y. Ming, Imperial College London, The Section of Infectious Diseases and Immunity, Commonwealth Building, Hammersmith Campus, London SW7 2AZ, UK; email: damien.ming@doctors.org.uk

\section{Tool for Eliminating Dog-Mediated Human Rabies through Mass Dog Vaccination Campaigns}

\author{
Eduardo A. Undurraga, ${ }^{1}$ Jesse D. Blanton, \\ S.M. Thumbi, Athman Mwatondo, \\ Mathew Muturi, Ryan M. Wallace
}

Author affiliations: Centers for Disease Control and Prevention, Atlanta, Georgia, USA (E.A. Undurraga, J.D. Blanton, R.M. Wallace); Kenya Medical Research Institute, Nairobi, Kenya (S.M. Thumbi); Washington State University, Pullman, Washington, USA (S.M. Thumbi); Kenya Ministry of Health, Nairobi (A. Mwatondo, M. Muturi)

DOI: https://doi.org/10.3201/eid2312.171148

The World Health Organization and collaborating agencies have set the goal of eliminating dog-mediated human rabies by 2030. Building on experience with rabies endemic countries, we constructed a user-friendly tool to help public health officials plan the resources needed to achieve this goal through mass vaccination of dogs.

$\mathrm{G}$ lobally, rabies kills $\approx 60,000$ persons annually; most $(\approx 99 \%)$ cases are transmitted by domestic dogs $(1-3)$. Controlling dog rabies through periodic mass vaccination campaigns substantially reduces human exposures (4). The elimination of dog rabies in most of the Western Hemisphere and countries in Asia has demonstrated the effectiveness and sustainability of vaccinating dogs $(5,6)$ by combining massive dog rabies vaccination with coordinated efforts of the medical and veterinary sectors (One Health approach), including education about responsible

${ }^{1}$ Current affiliation: Pontificia Universidad Católica de Chile,

Santiago, Chile. pet ownership, rabies awareness campaigns, and access to postexposure prophylaxis $(5,6)$.

The World Health Organization recommends that at least $70 \%$ of the dog population be vaccinated to control and potentially eliminate dog rabies (3). In 2016, WHO and partner organizations set the goal of eliminating dog-mediated human rabies by 2030 (7). This goal could be achieved by massive, costly administration of preexposure and postexposure prophylaxis, mass vaccination of dogs, or both.

Countries to which rabies is endemic in dogs are at different stages in their rabies control efforts $(5,8)$. Countries at early stages face barriers related to a limited understanding of the local epidemiology, logistic and operational challenges, competing priorities from other diseases, and lack of planning tools to reasonably project the resources needed. During 2016, we estimated the resources potentially required to eliminate dog rabies globally by 2030 (7). We combined multiple data sources to estimate 4 key factors that affect this goal: country development, cost of dog vaccination programs, potential demand for dog rabies vaccine, and estimated number of vaccinators. We aimed to realistically assess the global situation by highlighting the main challenges that might hamper elimination efforts. However, although these global estimates can inform an important discussion about global and regional strategic planning and resource mobilization, they are not necessarily useful to inform country-level decision-making. We addressed this limitation by providing a user-friendly tool that requires only limited country-specific data to help countries plan toward the goal of eliminating dog rabies through mass dog vaccination.

We designed the tool to plan for dog rabies elimination by 2030 (a 13-year framework). We assumed 13 years would be enough time for even the least developed rabies control programs to achieve elimination, provided the country was fully committed. The country's starting point within this time frame would depend on its current dog vaccination rate, and the given country would demonstrate incremental improvements in preparation for the vaccination campaign (e.g., training of workforce involved, dog population surveys) or in the proportion of the dog population vaccinated. The tool requires input of demographic data (human population, percentage urban, human-to-dog ratio); current dog vaccination coverage; logistic data for the campaigns (available vaccinators, dog vaccination rates, campaign duration); and an estimated cost per vaccinated dog. We constructed a worksheet to help users estimate the cost per vaccinated dog based on a pilot campaign. The tool (including assumptions, instructions, and data requirements) is available for public use (online Technical Appendix, https://wwwnc.cdc.gov/ EID/article/23/12/17-1148-Techapp1.xlsm) and already has been used in Haiti and Guatemala as part of a rabies elimination workshop held at the Centers for Disease Control and 

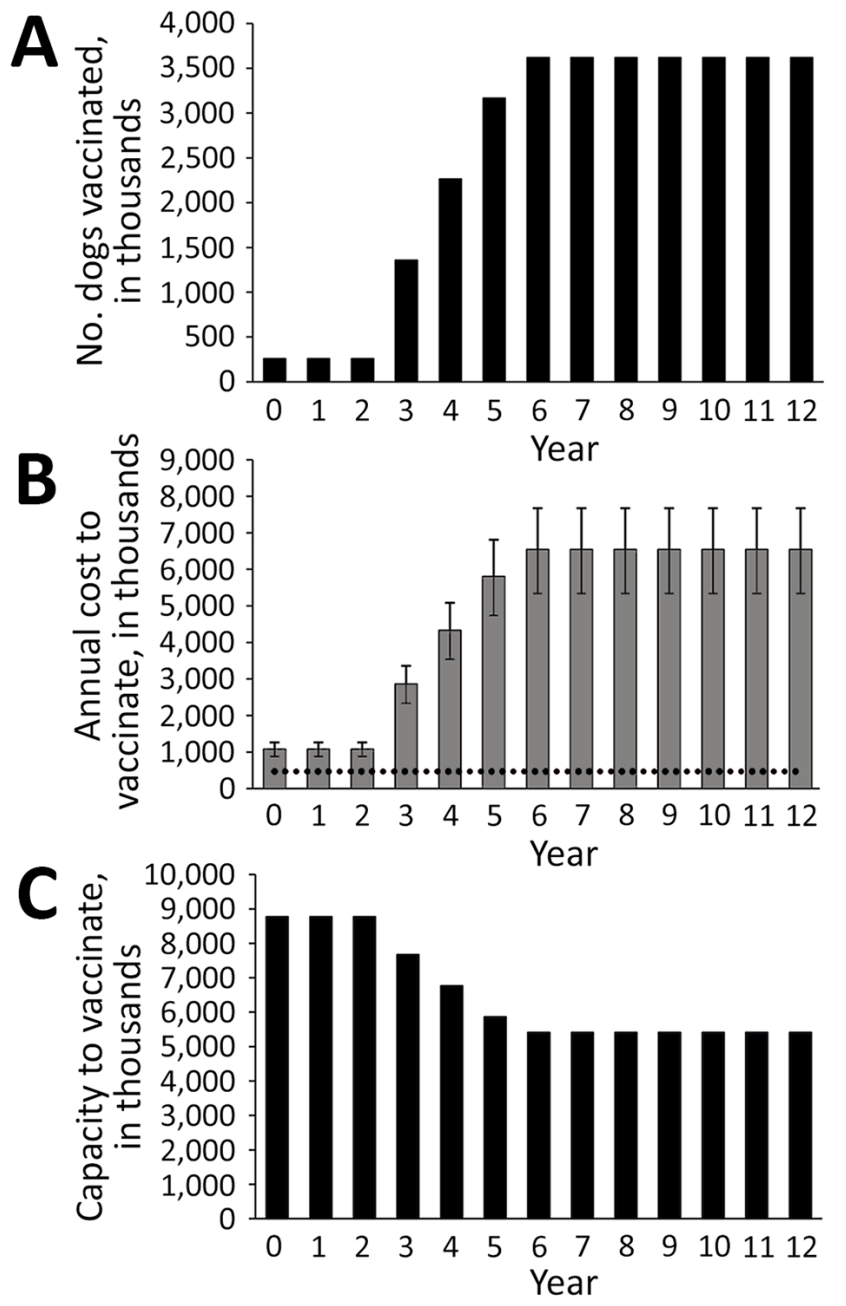

Figure. Illustrative results from the planning aid tool for controlling dog rabies through dog vaccination using input data, Kenya, 2016. A) Number of annual dog vaccinations required in accordance with World Health Organization recommendations. The tool assumes a threshold of $70 \%$ of dog vaccination during 7 years as a conservative estimate to eliminate dog-mediated rabies. The actual proportion of the dog population that needs to be vaccinated depends on local conditions of rabies transmission (10). Based on field data, Kenya estimates that 3 consecutive years of $70 \%$ coverage of dog vaccination would end dog-dog rabies transmission. The estimate by Hampson et al. (1) estimate of 523 annual deaths was based on active surveillance in eastern Kenya; current passive surveillance reports higher bite rates, so the estimate probably represents a lower bound of the number of deaths that could be avoided through mass dog vaccination. B) Total annual cost (US \$) of dog vaccination. Error bars indicate $95 \% \mathrm{Cls}$. Horizontal dotted line indicates current spending for dog vaccination. C) Net dog vaccination capacity (i.e., total number of dogs per year minus the number of dogs Kenya needs to vaccinate to achieve the dog vaccination coverage goal). If the number is positive, the country has enough capacity to vaccinate; if negative, the country needs more vaccinators, increased vaccination efficiency, or more campaign days. The estimated annual costs are based on the cost per dog vaccinated; excess vaccinator capacity is not included in the aggregate costs.
1Prevention (Atlanta, GA, USA). Users can account for uncertainty in point estimates by varying model parameters.

To show the utility of the planning tool, we input known information from 2 recent mass dog vaccination campaigns in western Kenya to project expected longterm costs and a timeline for rabies elimination. With a conservative estimate of 523 (95\% CI 138-1,100) annual human rabies-associated deaths and substantial medical costs (1), Kenya is actively trying to eliminate human rabies by 2030 (9). We used the following values: $46,050,302$ persons, of whom $26 \%$ live in urban areas; 7.4 and 21.2 humans per dog in rural and urban areas, respectively; $5 \%$ of the dog population vaccinated; 4,300 available vaccinators; 100 dogs vaccinated daily per vaccinator; a 21-day campaign in each region; an estimated cost per dog vaccinated of US \$1.81 (range US \$1.48-\$2.12); and 0\% discount rate (Figure). Conditional on the availability of resources, the results suggest that Kenya can eliminate dog rabies during the next 13 years, in line with the 2030 global goal; vaccination campaigns would cost $\approx$ US $\$ 62.0$ million (range US $\$ 50.6$ million- $\$ 72.8$ million), equivalent to $\approx \$ 56.0$ million additional aggregate spending over 13 years (not discounted). If the average vaccination rate was 100 dogs per vaccinator per day, Kenya would have enough capacity to conduct 21-day campaigns and reach the $70 \%$ dog vaccination goal.

The tool enables users to estimate the resources required to eliminate rabies using country-specific input values. We hope this tool will help stimulate and inform a necessary discussion on strategic planning, resource mobilization, and continuous execution of rabies elimination.

This work was funded by the Centers for Disease Control and Prevention.

Dr. Undurraga was a Steven M. Teutsch Prevention Effectiveness Fellow in the Division of Preparedness and Emerging Infections, National Center for Emerging and Zoonotic Infectious Diseases, Centers for Disease Control and Prevention, when this work was done. He is currently an assistant professor at the School of Government, Pontificia Universidad Católica de Chile, Santiago. His primary research interests include population health, health economics, and social policy.

\section{References}

1. Hampson K, Coudeville L, Lembo T, Sambo M, Kieffer A, Attlan M, et al.; Global Alliance for Rabies Control Partners for Rabies Prevention. Estimating the global burden of endemic canine rabies. PLoS Negl Trop Dis. 2015;9:e0003709. Erratum in: PLoS Negl Trop Dis. 2015;9:e0003786. http://dx.doi.org/10.1371/ journal.pntd.0003709

2. Knobel DL, Cleaveland S, Coleman PG, Fèvre EM, Meltzer MI, Miranda MEG, et al. Re-evaluating the burden of rabies in Africa and Asia. Bull World Health Organ. 2005;83:360-8. 
3. World Health Organization. WHO Expert Consultation on Rabies. Second report [cited 2016 Mar 30]. http://apps.who.int/iris/ bitstream/10665/85346/1/9789240690943_eng.pdf

4. World Health Organization. Rabies vaccines: WHO position paper. Wkly Epidemiol Rec. 2010;85:309-20 [cited 2017 Oct 5]. http://www.who.int/wer/2010/wer8532/en/

5. Rupprecht CE, Hanlon CA, Hemachudha T. Rabies re-examined. Lancet Infect Dis. 2002;2:327-43. http://dx.doi.org/10.1016/ S1473-3099(02)00287-6

6. Vigilato MAN, Clavijo A, Knobl T, Silva HMT, Cosivi O, Schneider MC, et al. Progress towards eliminating canine rabies: policies and perspectives from Latin America and the Caribbean. Philos Trans R Soc Lond B Biol Sci. 2013;368:20120143. http://dx.doi.org/10.1098/rstb.2012.0143

7. Wallace RM, Undurraga EA, Blanton JD, Cleaton J, Franka R. Elimination of dog-mediated human rabies deaths by 2030: needs assessment and alternatives for progress based on dog vaccination. Front Vet Sci. 2017;4:9. http://dx.doi.org/10.3389/fvets.2017.00009

8. Lembo T, Hampson K, Kaare MT, Ernest E, Knobel D, Kazwala RR, et al. The feasibility of canine rabies elimination in Africa: dispelling doubts with data. PLoS Negl Trop Dis. 2010;4:e626. http://dx.doi.org/10.1371/journal.pntd.0000626

9. Republic of Kenya Ministry of Health and Ministry of Agriculture. Strategic plan for the elimination of human rabies in Kenya 2014-2030 [cited 2017 Jun 1]. http://zdukenya.org/wp-content/ uploads/2012/09/National-Rabies-Elimination-Strategy.pdf

10. Zinsstag J, Dürr S, Penny MA, Mindekem R, Roth F, Menendez Gonzalez S, et al. Transmission dynamics and economics of rabies control in dogs and humans in an African city. Proc Natl Acad Sci U S A. 2009;106:14996-5001. http://dx.doi.org/ 10.1073/pnas.0904740106

Address for correspondence: Eduardo A. Undurraga, School of Government, Pontificia Universidad Católica de Chile, Vicuña Mackenna 4860, Macul CP 7820436, Región Metropolitana, Chile; email: eundurra@uc.cl

\section{Unexpected Infection with Armillifer Parasites}

\author{
Idzi Potters, Claude Desaive, \\ Steven Van Den Broucke, \\ Marjan Van Esbroeck, Lutgarde Lynen
}

Author affiliations: Institute of Tropical Medicine Antwerp, Antwerp, Belgium (I. Potters, S. Van Den Broucke, M. Van Esbroeck, L. Lynen); Central University Hospital of Liège, Liège, Belgium (C. Desaive)

DOI: https://doi.org/10.3201/eid2312.171189

Visceral pentastomiasis is usually found incidentally during surgery. We describe a case of visceral pentastomiasis discovered during inguinoscrotal hernia surgery for a man from Benin, Africa. Because surgical removal of nymphs is needed for symptomatic patients only, this patient's asymptomatic pentastomiasis was not treated and he recovered from surgery uneventfully.

$\mathrm{I}^{\mathrm{n}}$ n November 2015, a surgeon from Belgium, working for Medics without Vacation in Bassila, Benin, Africa, incidentally discovered pentastomiasis in an adult man during surgery for a massive inguinoscrotal hernia (half a liter content). Other than the hernia, the patient had no health problems. During the procedure, the surgeon observed at least 10 coiled, larva-like structures on the patient's peritoneal tissue. He removed the hernial sac and sent a tissue specimen to the national reference laboratory for parasitology at the Institute of Tropical Medicine (Antwerp, Belgium) for identification of the parasite. Apart from the hernia symptoms, the patient was asymptomatic, so the parasites were not removed; the patient's surgical recovery was uneventful.

Macroscopic examination of the peritoneal tissue detected 8 distinct, typical larva-like structures with an average length of 1-2 $\mathrm{cm}$ (Figure, panel A). Because the structures were suspected to be pentastomes, they were compared with reference material from the Institute of Tropical Medicine Educational Department (Figure, panel B) and confirmed as Armillifer spp. nymphs. On the basis of the patient's residence in Benin, and the fact that the recovered nymphs consistently exhibited $<22$ annuli, the structures were presumptively identified as $A$. armillatus $(1,2)$. Adults of this species are often found in the respiratory system of large snakes. Although no information was available regarding the patient's contact with snakes, the presence of this parasite in a resident of Benin is not surprising because snake consumption in that country is common practice. The patient's surgeon confirmed that dead snakes were indeed often sold for consumption along the streets near the hospital.

The Pentastomida are a peculiar group of gonochoric, vermiform endoparasites, currently classified as a unique phylum, related to branchiuran crustaceans (3). The main characteristics of this group of ancient parasitic arthropods are an often annulated elongate body and a mouth typically flanked by 2 pairs of hooks.

Human visceral pentastomiasis can be caused by several species of pentastomes: Linguatula serrata (worldwide, predominantly the Middle East), A. armillatus (West and Central Africa), A. moniliformis (Southeast Asia), A. grandis (Africa), A. agkistrodontis (China), Porocephalus crotali (worldwide, predominantly the Americas), and P. taiwana (China) $(2,4,5)$. Reported cases of human visceral pentastomiasis were caused mainly by $A$. armillatus pentastomes ( 6 ) from infected snakes, which shed ova in excretions and respiratory secretions, thereby 